\title{
Digestatos procedentes de la obtención de biogás a partir de purines vacunos en la calidad físico-química
} de un suelo árido

\section{Digestates from the production of biogas from cattle slurry in the physical-chemical quality of an arid soil}

\author{
Paul Gómez ${ }^{1}(\mathbb{D})$, Roxana Bardales ${ }^{2}$ (D), Omar Zeballos ${ }^{1 *}$
}

\section{RESUMEN}

El objetivo de esta investigación fue evaluar el efecto del biosol y del biol, en la calidad física y química del suelo en la Irrigación Majes. El biol y el biosol utilizados fueron los remanentes de la planta de Biogás de la Universidad Católica de Santa María, instalada en la Irrigación Majes. El experimento se realizó en una parcela experimental conducida bajo riego por goteo, estableciendo dos dosis de biosol, cuatro niveles de biol, y un tratamiento adicional, un testigo con fertilización química, teniendo un total de 9 tratamientos, distribuidos en un diseño de bloques completos al azar con arreglo factorial, y tres repeticiones. El biosol fue aplicado en fertilización de fondo antes de la instalación del experimento, mientras que las aplicaciones foliares del biol se realizaron al surco húmedo para los 15 , 30, 45 y 60 días después del trasplante. Las variables evaluadas fueron $\mathrm{pH}$, conductividad eléctrica (C.E.), potasio (K ppm), fósforo (P ppm), capacidad de intercambio catiónico (CIC), cationes cambiables, velocidad de infiltración y profundidad efectiva de las raíces. Se determinó que existe una tendencia a aumentar el pH y la C.E. cuando se realizan aplicaciones de digestatos, sin embargo el contenido de materia orgánica del suelo no varía significativamente. Las aplicaciones de digestatos contribuyeron a una mejora en la velocidad de infiltración.

Palabras claves: biol, biosol, zona árida, calidad de suelo.

\begin{abstract}
The objective of this research was to evaluate the effect of biosol and biol on the physical and chemical quality of the soil in Majes Irrigation. The biol and biosol used were the remnants of the Biogas plant of the Catholic University of Santa Maria, installed in Majes Irrigation. The experiment was carried out in an experimental plot conducted under drip irrigation, establishing two doses of biosol, four levels of biol, and an additional treatment, a control with chemical fertilization, having a total of 9 treatments, distributed in a design of complete blocks at random with factorial arrangement, and three repetitions. The biosol was applied as a background fertilization before the installation of the experiment, while the foliar applications of the biol were made to the wet furrow for 15, 30, 45 and 60 days after transplantation. The variables evaluated were $\mathrm{pH}$, electrical conductivity (EC), potassium (K ppm), phosphorus (P ppm), cation exchange capacity (CEC), changeable cations, infiltration rate and effective root depth. It was determined that there is a tendency to increase the $\mathrm{pH}$ and E.C. when digestate applications are made, however the organic matter content of the soil does not vary significantly. Digestate applications contributed to an improvement in the infiltration rate.
\end{abstract}

Keywords: liquid digestate, solid digestate; arid zones, soil quality.

\footnotetext{
${ }^{1}$ Universidad Católica de Santa María, Escuela de Posgrado, Arequipa, Perú

${ }^{2}$ Universidad Católica de Santa María, Facultad de Ciencias Farmacéuticas, Bioquímicas y Biotecnológicas, Arequipa, Perú

Autor de correspondencia. Email: omar_zc@hotmail.com
} 


\section{INTRODUCCIÓN}

La digestión anaeróbica (DA), es un proceso para convertir los desechos orgánicos en dos subproductos económicamente útiles: biogás, una fuente de energía renovable, y el digestato, cuyo uso se da en forma líquida y/o sólida (Koszel y Lorencowicz, 2015). En ese sentido la separación de digestatos crea dos productos, uno líquido y un material fibroso, conteniendo una gran cantidad de compuestos orgánicos de origen vegetal y microbiano y numerosos elementos minerales. Su característica clave, es una baja concentración de materia seca que varía desde un pequeño porcentaje hasta más del diez por ciento (Möller y Müller, 2012).

La composición mineral del digestato depende, en gran medida, de la composición del sustrato y del tipo de proceso de digestión (Albuquerque et al., 2012). Por lo tanto, el manejo del digestato líquido (biol) y del digestato solido (biosol) mediante su aplicación al suelo puede afectar de manera significativa sus propiedades. Durante la digestión anaerobia, la cantidad total de los nutrientes generalmente permanecen estables, pero otras características del sustrato de entrada pueden cambiar. Se ha demostrado que la relación de carbón y nitrógeno $\mathrm{C} / \mathrm{N}$ disminuye, mientras que el contenido de nitrógeno $(\mathrm{N})$ mineral $\left(\mathrm{NH}_{4}-\mathrm{N}\right)$ y el $\mathrm{pH}$ del sustrato pueden aumentar (Möller y Müller, 2012) Los digestatos provenientes de la DA se comportan como potenciales fertilizantes debido, a que la DA puede promover la preservación y acumulación de nutrientes inorgánicos como fósforo $(\mathrm{P})$, potasio $(\mathrm{K}) \mathrm{y}$ nitrógeno (N). (Pognani et al., 2009). Otros estudios sugieren que la aplicación directa de digestatos, como enmiendas del suelo, podría conllevar resultados conflictivos (Nkoa, 2014). Al respecto, Insam et al. (2015) sugiere que la concentración elevada de $\mathrm{N}$ en los digestatos puede acelerar la mineralización del $\mathrm{C}$ (priming effect). Por otro lado, Smith et al. (2014) señalan que el carbono proveniente de la DA es más estable que el de otros desechos orgánicos. y que la materia orgánica contenida en los digestatos tiene un gran potencial para aumentar el secuestro de carbono en el suelo.
El efecto esperado por la aplicación de digestatos en la producción de cultivos, es motivo de controversia, porque es el resultado de numerosos procesos que afectan la relación suelo/planta. El primero de ellos es el referido al contenido de elementos minerales, el cual es muy variable debido a la composición natural de la materia prima. Acorde a Gutser et al. (2005), el valor fertilizante del digestato es comparable a los excrementos secos de aves de corral. El segundo criterio en la evaluación del digestato, está relacionado con su impacto en la salud del suelo. Este término comprende una amplia gama de funciones del suelo, como transformación de carbono, ciclo de nutrientes, mantenimiento de la estructura del suelo y control de plagas y enfermedades. Todos estos procesos determinan el estado actual de la productividad del suelo (Kibblewhite et al., 2007)

El digestato, como fertilizante, no solo aporta N, sino muchos otros elementos, como nutrientes y metales pesados (Barabasz et al., 2002). El impacto del digestato en la biodisponibilidad de un elemento en particular, está relacionado principalmente con la concentración o la cantidad de nutrientes incorporados al suelo (Koszel y Lorencowicz, 2015 y Nkoa, 2014). Estudios a corto plazo han demostrado que la aplicación de digestatos anaeróbicos en los suelos, pueden tener efectos positivos en sus propiedades físicas, como reducción de la densidad aparente, aumento de la saturación hidráulica, conductividad y mejora de la capacidad de retención de humedad (Nkoa, 2014).

La calidad del suelo es una característica compleja, determinada por componentes físicos, químicos y biológicos. Al respecto Doran y Parkin (1994) definen la calidad del suelo, como la capacidad de un suelo para mantener la productividad biológica, mantener la calidad ambiental y promover la salud de las plantas y los animales. Los cambios en la comunidad microbiana del suelo, pueden ocurrir más rápidamente que los cambios en otras características del suelo y, por lo tanto, se cree que los procesos microbianos del suelo son indicadores sensibles de los cambios en la calidad del suelo (Kennedy y Papendick, 1995). 
A pesar de los extensos estudios sobre la composición elemental de los digestatos provenientes de plantas de biogás, el conocimiento de su impacto en los procesos del suelo, aunque decisivo para el estado de su fertilidad y la respuesta de los cultivos, aún es deficiente (Przygocka-Cyna y Grzebisz, 2018). Es por ello, que el objetivo de esta investigación fue evaluar el efecto de dos dosis de biosol (digestato solido) y de cuatro niveles de biol (digestato líquido) en la calidad física y química de suelo en zonas áridas.

\section{MATERIALES Y MÉTODOS}

Se utilizó un diseño de bloques completos al azar con nueve tratamientos y tres repeticiones, conducidos bajo un arreglo factorial (2A4B), y un testigo (tratamiento adicional), que tuvo solo fertilización química. El factor digestato solido (biosol) contemplaba dos niveles, 2 y 4 t/ha. Mientras que el factor digestato liquido (biol), contemplaba cuatro niveles, $20 \%, 35 \%$, $50 \%$ y $60 \%$ ( $\%$ de biol puro mezclado con agua). Para el tratamiento adicional (ADC) se utilizó urea, fosfato diamónico y cloruro de potasio. Los digestatos provienen de los remanentes de la producción de biogás, y su purificación a biometano mediante "reformado" concentrado y purificado del metano $\left(\mathrm{CH}_{4}\right)$ de la mezcla gaseosa por arriba del 95\%, ofreciendo claras ventajas y oportunidades para su uso con respecto al biogás precursor, ya que puede ser presurizado y envasado a alta y baja presión (Reátegui et al., 2018).

La prueba estadística utilizada para los diferentes cuadrados medios asociados a sus fuentes de variabilidad, fue la F de Fisher. Para comparar las medias, se utilizó la prueba de Duncan al 0,05 de significancia, y para comparar los tratamientos en estudio con el tratamiento adicional se utilizó contrastes ortogonales. La unidad experimental tuvo una área de $20 \mathrm{~m}^{2}$, el largo del campo experimental fue de $40 \mathrm{~m}$ y el ancho del campo experimental de $17 \mathrm{~m}$, teniendo una área total experimental de $680 \mathrm{~m}^{2}$.

El experimento se instaló en el fundo "La Católica", propiedad de la Universidad Católica de Santa María (UCSM) localizado en la Irrigación Majes, el cual se encuentra comprendido dentro de las coordenadas $16^{\circ}$ $15^{\prime}$ latitud Sur y $72^{\circ} 15^{\prime}$ de longitud Oeste y a una altitud promedio de 1,375 m.m.s.m., a una distancia de $100 \mathrm{~km}$ de la ciudad de Arequipa, con dirección NorOeste (AUTODEMA, 2017). La ampliación de la frontera agrícola por irrigación (Irrigación Majes) se da en tablazos costeros, prácticamente sin precipitaciones, constituyendo uno de los desiertos más áridos del planeta (Jiménez et al., 2002). Estas condiciones hacen que sea considerado una zona árida.

El experimento se condujo en un suelo de textura francoarenosa, de la costa sur del Perú, y se usó como plantas indicadora a la cebolla (Allium cepa), cuyo manejo fue bajo la misma metodología del agricultor, es decir, forma de siembra, riego (goteo), aplicación de materia orgánica (estiércol de vacuno 20 t/ha) y labores culturales complementarias. El cultivar de cebolla utilizado fue "Roja camaneja" en la preparación del terreno. Como primer paso se procedió al desempiedre y limpieza de malezas y residuos de la campaña anterior. Posteriormente se incorporó estiércol de vacuno, junto con aplicaciones periódicas de riego pesado por espacio de 1 mes, para después aplicar los niveles de biosol acorde a los tratamientos en estudio, introduciéndolo con disco dentro de la capa arable. Luego se nieveló con riel, de tal forma que quede plano o nivelado dejando apto el terreno para el surcado y posterior trasplante. Las aplicaciones foliares de biol se realizaron al surco húmedo en Drench, para el crecimiento vegetativo y llenado del bulbo, con 4 aplicaciones, 15 , 30, 45 y 60 días después del trasplante. El biol y biosol fueron provistos por el fundo de la Universidad Católica de Santa María, provenientes de su planta de producción de biogás. Se realizaron análisis microbiológicos y de nutrientes del biol producido en dicha planta (Tabla 1 y Tabla 2). Al final del ciclo productivo del cultivo se tomaron muestras de suelo de cada unidad experimental, para luego realizar análisis de caracterización de dichas muestras. Al final del periodo vegetativo del cultivo se determinó la profundidad efectiva de raíces, midiendo la longitud $(\mathrm{cm})$ que estas alcanzaron, tomando muestras aleatorias en cada unidad expe- 
rimental. Se utilizó el método de los cilindros infiltrómetros, para evaluar la velocidad de infiltración en cada unidad experimental, siendo el método usado donde se establecen sistemas de riego por faja, compartimentos cerrados, aspersión y goteo (Fuentes, 1998).

Tabla 1. Análisis microbiológico del biol aplicado en el experimento

\begin{tabular}{ccccc}
\hline & OMT & & BFNVL & BN \\
Bacterias (UFC/ml) & Actinomicetos (UFC/ml) & Hongos (UFC/ml) & $\begin{array}{c}\text { BFrganismos/ml) } \\
\text { (Organismos/ml) }\end{array}$ \\
\hline $4,93 \times 10^{5}$ & $3,00 \times 10^{3}$ & $1,70 \times 10^{2}$ & $7,50 \times 10^{1}$ & $4,30 \times 10^{4}$ \\
\hline
\end{tabular}

$\mathrm{UFC}=$ Unidad formadora de colonia; OMT = Organismos mesófilos totales; $\mathrm{BFNVL}=$ Bacterias fijadoras de $\mathrm{N}$ de vida libre; $\mathrm{BN}=\mathrm{Bacterias}$ nitrificantes.

Tabla 2. Análisis de nutrientes del biol aplicado en el experimento

\begin{tabular}{cc}
\hline Parámetro & Valor \\
\hline pH & 7,83 \\
C.E. $(\mathrm{dS} / \mathrm{m})$ & 5,60 \\
Solidos totales $(\mathrm{g} / \mathrm{L})$ & 4,96 \\
M.O. en Solución $(\mathrm{g} / \mathrm{L})$ & 1,90 \\
Ntotal $(\mathrm{mg} / \mathrm{L})$ & 364,00 \\
Ptotal $(\mathrm{mg} / \mathrm{L})$ & 73,24 \\
K total $(\mathrm{mg} / \mathrm{L})$ & 810,00 \\
Catotal $(\mathrm{mg} / \mathrm{L})$ & 159,50 \\
Mgtotal $(\mathrm{mg} / \mathrm{L})$ & 147,50 \\
Natotal $(\mathrm{mg} / \mathrm{L})$ & 505,00 \\
Fetotal $(\mathrm{mg} / \mathrm{L})$ & 4,01 \\
Cutotal $(\mathrm{mg} / \mathrm{L})$ & 0,76 \\
Zntotal $(\mathrm{mg} / \mathrm{L})$ & 1,58 \\
Mntotal $(\mathrm{mg} / \mathrm{L})$ & 0,80 \\
B total $(\mathrm{mg} / \mathrm{L})$ & 2,33 \\
\hline
\end{tabular}

M.O. = Materia orgánico; $\mathrm{N}=$ Nitrógeno, $\mathrm{P}=$ Fósforo $\mathrm{K}=$ Potasio $\mathrm{Ca}=$ Calcio $; \mathrm{Mg}=$ Magnesio $; \mathrm{Na}=$ Sodio $; \mathrm{Fe}=$ Hierro $; \mathrm{Cu}=$ Cobre $;$ $\mathrm{Zn}=\mathrm{Zinc} ; \mathrm{Mn}=$ Manganeso $; \mathrm{B}=$ Boro

\section{RESULTADOS Y DISCUSIÓN}

En la Tabla 3, se observa que existe diferencias estadísticas significativas para la fuente de variación del factor bloque en las variables de $\mathrm{pH}, \mathrm{K}$ (ppm) y K (meq/100 g). Para el factor biosol se observa que existe diferencia estadística significativa para la variable C.E., mientras que para el factor biol y la interacción biol X biosol se observa que no existen diferencias estadísticas significativas en todas las variables evaluadas. Para la comparación Factorial Vs Adicional se observa que existe diferencias estadísticas significativas para las variables de $\mathrm{pH}$ y $\mathrm{Ca}(\mathrm{meq} / 100 \mathrm{~g})$, asimismo los coeficientes de variabilidad en todas las variables evaluadas a excepción de P muestran estar dentro de lo aceptable.

En la tabla 4 se observa que a un mayor nivel de biosol (4 t/ha) aumento de manera significativa la C.E. del suelo, lo que sería perjudicial para el desarrollo de los cultivos en dichos suelos.

Tabla 3 Análisis de varianza para las propiedades químicas del suelo

\begin{tabular}{|c|c|c|c|c|c|c|c|c|c|c|c|c|c|}
\hline \multirow{2}{*}{ F.V } & \multirow{2}{*}{ G.L } & \multicolumn{11}{|c|}{ F.C } & \multirow{2}{*}{$\begin{array}{c}\text { F.T. } \\
(\alpha=0,05)\end{array}$} \\
\hline & & pH & C.E & $\% \mathrm{CaCO}_{3}$ & $\%$ MO & $\mathbf{P}$ & $\mathbf{K}^{1}$ & CIC & $\mathrm{Ca}$ & Mg & $\mathbf{K}^{2}$ & $\mathrm{Na}$ & \\
\hline Bloque & 2 & $29,97 *$ & 1,53 & 2,73 & 1,46 & 0,34 & $4,36 *$ & 0,36 & 0,92 & 0,46 & $5,67 *$ & 3,63 & 3,63 \\
\hline Biosol & 1 & 0,49 & $5,96 *$ & 0,00 & 0,52 & 0,01 & 2,73 & 0,17 & 0,52 & 0,02 & 0,38 & 4,49 & 4,49 \\
\hline Biol & 3 & 2,59 & 0,56 & 0,84 & 1,27 & 0,32 & 0,34 & 0,34 & 0,41 & 0,16 & 1,04 & 3,24 & 3,24 \\
\hline Biosol X Biol & 3 & 0,73 & 0,31 & 0,24 & 1,68 & 1,50 & 1,08 & 1,03 & 0,51 & 1,64 & 0,50 & 3,24 & 3,24 \\
\hline $\begin{array}{c}\text { Factorial Vs } \\
\text { Adicional }\end{array}$ & 1 & $8,01 *$ & 0,01 & 2,45 & 0,76 & 0,01 & 3,43 & 2,35 & $4,77 *$ & 0,01 & 2,28 & 4,49 & 4,49 \\
\hline $\begin{array}{c}\text { Error } \\
\text { experimental } \\
\end{array}$ & 16 & & & & & & & & & & & & \\
\hline C.V & & 1,06 & 7,01 & 14,48 & 14,55 & 45,29 & 28,42 & 12,26 & 15,04 & 16,75 & 24,46 & 39,8 & \\
\hline
\end{tabular}

${ }^{1} \mathrm{ppm} ;{ }^{2} \mathrm{meq} / 100 \mathrm{~g}$

Tabla 4. Efecto principal del factor Biosol en promedio de los niveles del factor Biol para C.E

\begin{tabular}{lcc}
\hline & Promedio C.E. & Significancia * \\
\hline $\begin{array}{l}\text { Biosol } \\
\mathbf{t} \text { t/ha }\end{array}$ & 2,46 & $\mathrm{a}$ \\
$\begin{array}{l}\text { Biosol } \\
\mathbf{4} \text { tha }\end{array}$ & 2,64 & $\mathrm{~b}$ \\
\hline
\end{tabular}

En la tabla 5, se observa que para las variables $\mathrm{pH}$ y $\mathrm{K}$ (ppm) existio una tendencia a aumentar su valor para todos los tratamientos evaluados en relación al tratamiento adicional (sin biol ni biosol), para la C.E se observa la misma tendencia pero solo con los tratamientos con aplicaciones de biosol de $4 \mathrm{t} / \mathrm{ha}$. 
Tabla 5. Promedios de las variables evaluadas.

\begin{tabular}{cccccccccccc}
\hline Tratamiento & $\mathbf{p H}$ & $\mathbf{C . E}$. & $\mathbf{\% C a C O 3}$ & $\mathbf{\% M . O}$ & $\mathbf{P}$ & $\mathbf{K}^{\mathbf{1}}$ & $\mathbf{C I C}$ & $\mathbf{C a}$ & $\mathbf{M g}$ & $\mathbf{K}^{\mathbf{2}}$ & $\mathbf{N a}$ \\
\hline ADC & 7,28 & 2,56 & 1,57 & 0,93 & 20,17 & 224,67 & 7,73 & 5,83 & 0,67 & 0,97 & 0,26 \\
B2B20 & 7,35 & 2,47 & 2,13 & 0,79 & 17,57 & 304,33 & 6,51 & 4,60 & 0,61 & 1,15 & 0,15 \\
B2B35 & 7,44 & 2,51 & 2,20 & 0,87 & 17,57 & 329,00 & 6,56 & 4,66 & 0,64 & 1,14 & 0,12 \\
B2B50 & 7,41 & 2,33 & 1,73 & 0,75 & 30,30 & 351,67 & 6,83 & 4,54 & 0,74 & 1,36 & 0,17 \\
B2B65 & 7,41 & 2,53 & 2,43 & 0,67 & 18,13 & 440,00 & 7,52 & 5,10 & 0,70 & 1,50 & 0,23 \\
B4B20 & 7,37 & 2,68 & 2,37 & 1,15 & 24,00 & 308,33 & 7,52 & 5,37 & 0,74 & 1,19 & 0,22 \\
B4B35 & 7,52 & 2,65 & 2,07 & 0,67 & 18,23 & 306,00 & 6,88 & 4,95 & 0,68 & 1,10 & 0,15 \\
B4B50 & 7,44 & 2,61 & 1,90 & 0,69 & 16,17 & 297,67 & 6,77 & 4,59 & 0,64 & 1,39 & 0,15 \\
B4B65 & 7,36 & 2,62 & 2,20 & 0,90 & 23,33 & 271,67 & 6,83 & 4,85 & 0,60 & 1,17 & 0,21 \\
\hline
\end{tabular}

${ }^{1} \mathrm{ppm} ;{ }^{2} \mathrm{meq} / 100 \mathrm{~g}$

La tendencia a aumentar el $\mathrm{pH}$ del suelo y las diferencias en cuanto a la $\mathrm{CE}$, podría deberse al carácter alcalino del digestato en sí mismo, ocasionado por su alto contenido de amonio (Voelkner et al., 2015). Sin embargo, un aumento del contendido de amonio no garantiza una mejora en la utilización eficiente del $\mathrm{N}$ (Möller y Müller, 2012). Con respecto al \% M.O., este no tuvo mayores cambios en los tratamientos con aplicaciones de digestatos en relación al tratamiento adicional, evidenciando que la aplicación de digestatos no mejoró significativamente el contendido de M.O del suelo, lo cual coincide con lo reportado con Šimon et al., (2015). La disminución del contenido de
$\mathrm{Ca}$ en los tratamientos que contienen digestatos, en relación al tratamiento adicional, se debió principalmente a que las aplicaciones de disgestatos propiciaron a que haya una cristalización parcial del $\mathrm{Ca}$ en carbonatos y fosfatos (Möller y Müller, 2012; Marcato et al., 2008)

En la tabla 6, se puede observar que no existe diferencias estadísticas significativas para todas las variables evaluadas. Para la comparación Factorial Vs Adicional, se observa que existen diferencias estadísticas significativas, asimismo el coeficiente de variabilidad muestra estar dentro de lo aceptable.

Tabla 6.Análisis de varianza para velocidad de infiltración

\begin{tabular}{ccccccc}
\hline F.V & G.L & S.C & C.M & F.C & F.T & Sig. \\
\hline Bloque & 2 & 4,2330 & 2,1165 & 0,1754 & 3,6337 & 4,4940 \\
Biosol & 1 & 6,4170 & 6,4170 & 0,5319 & 3,2389 & N.S \\
Biol & 3 & 29,4454 & 9,8151 & 0,8136 & 3,2389 & N.S \\
Biosol X Biol & 3 & 44,5194 & 14,8398 & 1,2302 & 4,4940 \\
Factorial Vs & 1 & 68,8509 & 68,8509 & 5,7075 & & \\
Adicional & 16 & 193,0131 & 12,0633 & & \\
Error experimental & 29,98 & & &
\end{tabular}

En la tabla 7, se observa que existe una tendencia a aumentar la velocidad de infiltración conforme se aplicaron los niveles de biol y biosol, en relación al tratamiento adicional. Más aun, esto se evidencia al encontrar que en dicho contraste (tabla 6) se encontró diferencias estadísticas significativas.

Esta tendencia a incrementar la velocidad de infiltración, se debe al incremento de materia orgánica contendida en los digestatos. Al respecto, Wolf y Snyder
Tabla 7. Promedios de velocidad de infiltración $(\mathrm{cm} / \mathrm{h})$

\begin{tabular}{cc}
\hline Tratamiento & Velocidad de infiltración $\mathbf{( c m} / \mathbf{h})$ \\
\hline B4B35 & 14,7258439 \\
B2B65 & 14,2726968 \\
B4B50 & 12,9666339 \\
B2B50 & 12,5981991 \\
B4B65 & 12,5337419 \\
B2B20 & 10,4718396 \\
B4B20 & 10,427628 \\
B2B35 & 9,17534264 \\
ADC & 8,25776568 \\
\hline
\end{tabular}


(2003), señalan que la lenta permeabilidad de los suelos mejora con una mejor estructura de los mismos, lo cual, se logra con aplicaciones de materia orgánica coincidiendo con lo reportado por Garg et al. (2015), quienes mostraron que la aplicación al suelo de digestatos líquidos, reduce la densidad aparente e incrementa la conductividad hidráulica saturada.

Con respecto a la profundidad efectiva de raíces, no se evidencio diferencias estadísticas significativas. Esto se debe, a que no hubo ningún cambio significativo en las unidades experimentales en lo referente a algunas propiedades físicas del suelo, como textura y estructura. En tal sentido, no hubo mayor resistencia a la penetración de las raíces al suelo (FAO, 2000) estando sus valores dentro de lo normal acorde a los señalado por FAO (2006)

\section{CONCLUSIONES}

Existe una tendencia a aumentar el $\mathrm{pH}$ y la C.E. del suelo, cuando se realizan aplicaciones de biol y biosol. Debiendo tener especial cuidado con aplicaciones de biosol por encima de $4 \mathrm{t} / \mathrm{ha}$, ya que podría perjudicar la productividad de los cultivos. No se evidencio una mejora significativa en el contenido de M.O de los suelos después de la aplicación de los tratamientos que contenían digestatos.

La aplicación de biol y biosol, mejora la velocidad de infiltración del suelo evidenciados en un efecto positivo en el suelo debido a una mayor circulación del agua.

\section{V.AGRADECIMIENTOS}

Esta investigación fue financiada gracias a la subvención proveniente del fondo para la investigación en la Universidad Católica de Santa María (Resolución 24155-2017), concurso organizado por el Vicerrectorado de Investigación.

\section{REFERENCIAS BIBLIOGRÁFICAS}

Alburquerque, J. A., C. de la Fuente, A. Ferre-Costa, L. Carrasco, J. Cegarra, M. Abad y M. P. Bernal. 2012. "Assessment of the fertiliser potential of digestates from farm and agro- industrial residues.” Biomass Bioenergy 40: 181-189.

AUTODEMA(Autoridad Autónoma de Majes). 2017. Proyecto Especial Majes Siguas 2002. https://www.autodema.gob.pe/historia/(Consultada 15 de noviembre 2017).

Barabasz, W., D. Albińska, M. Jaśkowska y J. Lipiec. 2002. "Ecotoxicoilogy of aluminium." Pol J Environ Studies 193: 199-203.

Doran, J. W. y T. P. Parkin. 1994. "Defining and assessing soil quality”. En Defining soil quality for a sustainable environment. Doran, J. W., D. C. Coleman, D. F. Bezdicek, y B. A. Stewart (eds). Wisconsin (EEUU): American Society of Agronomy Special Publication

FAO (Organización de las Naciones Unidas para la Agricultura y la Alimentación). 2000. Los principales factores ambientales y de suelos que influyen sobre la productividad y manejo. Roma (Italia): FAO.

FAO (Organización de las Naciones Unidas para la Agricultura y la Alimentación). 2006. Evapotranspiración del cultivo: Guías para la determinación de los requerimientos de agua de los cultivos. Roma (Italia): FAO.

Fuentes, J. L. 1998. Técnicas de riego. Madrid (España): Mundi-Prensa.

Garg, R. N., H. Pathak, D. K. Das y R.K. Tomar. 2005. "Use of flyash and biogas slurry for improving wheat yield and physical properties of soil." Environmental Monitoring and Assessment 107: 1-9.

Gutser, R., T. Ebertseder, A. Weber, M. Schraml y U. Schmidhalter. 2005. "Short-term and residual availability of nitrogen after long-term application of organic fertilizers on arable land." $J$ Plant Nutr Soil Sci 168: 439-446. DOI: 10.1002/jpln.200520510

Insam, H., M. Gomez-Brandon y J. Ascher. 2015. "Manure-based biogas fermentation residues - friend or foe of soil fertility?" Soil Biol Bioche $h \quad 84: 1-14$. D O I : 
10.1016/j.soilbio.2015.02.006

Jimenez, P., J. Villasante, C. Bernabe y L. Villegas.2 002. "Ecosistemas de Arequipa. Oferta ambiental y desarrollo sostenible". Zonas Aridas 7 (1): 118-132.

Kennedy, A. C., y R. I Papendick. 1995. "Microbial characteristics of soil quality". Journal of Soil and Water Conservation 50: 243-248.

Kibblewhite, M. G., K. Titz y M. J. Swift. 2007. "Soil health in agricultural systems". Philos Trans $R$ Soc Lond B Biol Sci 363: 685-701.

Koszel, M. y E. Lorencowicz. 2015. “Agricultural use of biogas digestate as a replacement fertilizers." Agriculture and Agricultural Science Procedia 7: 119-124. D O I : 10.1016/j.aaspro.2015.12.004

Marcato, C. E., E. Pinelli, P. Pouech, P. Winterton y M. Guiresse. 2008. "Particle size and metal distributions in anaerobically digested pig slurry". Biores. Technol. 99: 2340-2348.

Möller, K. y T. Müller. 2012. “Effects of anaerobic digestion on digestate nutrient availability and crop growth: A review." Engineering in Life Sciences 12 (3): 242-257.

Nkoa, R. 2014. “Agricultural benefits and environmental risks of soil fertilization with anaerobic digestates: a review." Agron. Sustainable Dev 34: 473-492

Pognani, M., G. D'Imporzano, B. Scaglia, y F. Adani. 2009. "Substituting energy crops with organic fraction of municipal solid waste for biogas production at farm level: a full-scale plant study." Process Biochem. 44: 817-821.

Przygocka-Cyna, K., y W. Grzebisz. 2018. "Biogas digestate - benefits and risks for soil fertility and crop quality - an evaluation of grain maize response". Open Chemistry 16: 258-271.

Reátegui, J., J. Peña, L. Cárdenas, J. Castro, F. Mejía, S. Mestas, y F. Roque. 2018. Manual de producción y uso de biometano presurizado a baja y alta presión. Arequipa (Perú): Univer- sidad Católica de Santa María.

Šimon, T., E. Kunzová, y M. Friedlová. 2015. “The effect of digestate, cattle slurry and mineral fertilization on the winter wheat yield and soil quality parameters." Plant Soil Environ. 61: 522-527.

Smith, J., A. Abegaz, R .B. Matthews, M. Subedi, E. R. Orskov, V. Tumwesige, y P. Smith. 2014. "What is the potential for biogas digesters to improve soil fertility and crop production in Sub-Saharan.” Africa Biomass Bioenergy 70: $58-72$.

Voelkner, A., S. Ohl, D. Holthusen, E. Hartung, E. Dörner, y R. Horn. 2015. "Impact of mechanically pre-treated anaerobic digestates on soil properties." Journal of Soil Science and Plant Nutrition 15 (4): 882-895.

Wolf, B. y G. H. Snyder. 2003. Sustainable Soils: The place of organic matter in sustaining soils and their productivity. Nueva York (USA): Food Products Press 